\title{
Zebrafish: a new model system for the development of safe opioid therapies
}

Zaig, S. et al. elife 10, e63407 (2021)

Opioids are widely used for pain management, but these drugs have severe adverse effects, including opioid-induced respiratory depression (OIRD). OIRD is caused by the activation of $\mu$-opioid receptors (MOR) expressed on the surface of neurons in brainstem respiratory centers, which depresses breathing and can be lethal.

Naloxone is a MOR blocker that can be used to reverse OIRD in patients, but its blocking action also reduces analgesia. Safer pain therapies are needed, but the lack of animal models allowing high-throughput screening approaches has hindered the discovery of novel opioids that don't cause OIRD and/or pharmacotherapies that block OIRD without affecting analgesia.

In a new study in eLife, a team of investigators led by Gaspard Montandon at the University of Toronto used behavioral assays to study the effects of opioids on nociception and respiratory rate in zebrafish larvae. Their findings suggest that opioid pharmacology is preserved in zebrafish and support the use of zebrafish to test the analgesic properties and safety of opioid drugs. "Our assays provide simple, yet effective, ways to quickly test potential drug candidates in a complex animal model while preserving its central nervous system and brain blood barrier," write the investigators.

First, the team analyzed the effects of opioid administration on fish breathing. Given that zebrafish use gills to breathe and their mouth to force water across the gill surface, the researchers used the rate of mandible movements as an index of respiratory rate. They recorded mandible movements with a high-definition camera positioned on top of zebrafish exposed to embryo medium (control), fentanyl alone or in combination with naloxone. Quantification of mandible movements by looking at pixel changes in the region of the mandible showed that fentanyl induced respiratory rate depression, which was reversed by naloxone.
Next, the team used a model of nociception combining exposure to a nociceptive stimulus such as (allyl)-isothiocyanate (AITC) and measurements of the subsequent swimming escape response to determine the analgesic properties of fentanyl. Analysis of swimming behavior revealed that AITC increased swimming velocity, an effect reduced by fentanyl, which was reversed by naloxone.

The study also shows that the two major strains of wild-type zebrafish, $A B$ and Tübingen showed different sensitivities to fentanyl. "This strain-specificity suggests that the mechanisms of action of opioid drugs differ between zebrafish strains which could be due polymorphisms of the MOR gene as it can be found in humans or genetic differences in various genes involved in MOR inhibition," write the investigators.

Alexandra Le Bras

Published online: 26 April 2021 https://doi.org/10.1038/s41684-021-00763-6

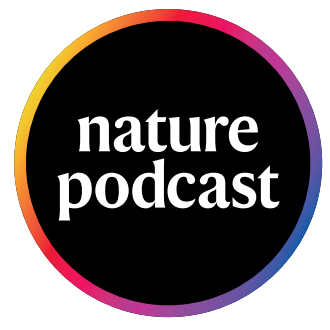

\section{The week's best science, from the world's leading science journal.}

NATURE.COM/NATURE/PODCAST 